Nervenarzt $2010 \cdot 81: 1279-1280$ DOI 10.1007/s00115-010-3048-y Online publiziert: 23. Oktober 2010 (c) Springer-Verlag 2010

F. Schneider ${ }^{1} \cdot$ W. Maier ${ }^{2}$

${ }^{1}$ Klinik für Psychiatrie und Psychotherapie, RWTH Aachen University, Aachen

${ }^{2}$ Klinik und Poliklinik für Psychiatrie und Psychotherapie, Universitätsklinikum Bonn

\title{
Interdisziplinäre Psychiatrie
}

Psychische Krankheiten betreffen den ganzen Menschen: Psyche und Körper, Seele und Gehirn, individuelle genetische Anlage und Erfahrung, Lebensgeschichte und Zukunftspotenziale. Das Menschenbild der Psychiatrie ist also im umfassenden Sinn ganzheitlich. Die wissenschaftliche Orientierung des Fachs kann sich somit nicht nur auf den engeren klinischen, diagnostisch-therapeutischen Kernbereich (wie Psychopathologie, Psychotherapie, Psychopharmakologie, Neuropsychiatrie und Psychosomatik) begrenzen. Die Entwicklungstendenzen und Möglichkeiten klinischer Nachbardisziplinen, methodischer Querschnittsfächer, Lebenswissenschaften insgesamt und Kulturwissenschaften sowie von Psychologie und Rechtswissenschaften eröffnen auch für die Erforschung psychischer Krankheiten und deren optimale Behandlung Potenziale und Perspektiven. Diesem großen Thema widmet sich der DGPPNKongress 2010 unter dem Motto „Psychiatrie interdisziplinär". Dieses Heft bietet eine hoffentlich spannende, begrenzte Auswahl von zukunftsweisenden, hoffnungsvollen und kritischen Perspektiven zu diesem Thema.

\section{(7) Das Menschenbild der Psychiatrie ist im umfassenden Sinn ganzheitlich}

Unser Ziel ist es, Ihnen einen Überblick über die verschiedenen Überschneidungsbereiche der Psychiatrie mit den Nachbardisziplinen zu geben, wobei aus Platzgründen nur einige Bereiche angeschnitten werden können.
Wolfgang Oertel und Tilo Kircher stellen im ersten Beitrag dieses Hefts das Verhältnis zwischen Psychiatrie und Neurologie heraus. Dabei berichten sie zunächst über aktuelle Entwicklungen in Klinik und Forschung getrennt für beide Fächer, bevor, am Beispiel der Demenz, auf gemeinsame Ansätze eingegangen wird. Die Autoren betonen dabei auch die Relevanz interdisziplinärer Methodenkompetenz und Infrastruktur für eine erfolgreiche Zusammenarbeit, besonders im Bereich der Forschung sowie die Bedeutung der gemeinsamen Aus- und Weiterbildung.

Der Beitrag von Anja Schneider, Peter Falkai und Andreas Papassotiropoulos, der im Bereich der molekularen Psychologie und der Schnittstelle zur Biologie angesiedelt ist, behandelt das Thema der Pathophysiologie von Demenzen. Die Autoren beschreiben auf molekularer Ebene verschiedene Mechanismen, die gegen Tau-vermittelte Toxizität bei der Alzheimer-Erkrankung wirken könnten und im Rahmen einer kausalen Behandlung von Alzheimer in Zusammenarbeit der verschiedenen beteiligten Teildisziplinen weiter erforscht werden sollten.

Anschließend beschäftigen sich Beate Herpertz-Dahlmann und Sabine Herpertz mit den Verknüpfungen zwischen Kinder- und Jugendpsychiatrie und Psychiatrie. Die Autorinnen berichten zunächst über die historische Entwicklung der Kinder- und Jugendpsychiatrie als eigenständige Fachdisziplin. Das Manuskript beinhaltet auch sehr konkrete Beschreibungen, an welchen Stellen eine vertiefte Kooperation zwischen den bei- 
den „Schwester“-Disziplinen wichtig und sinnvoll ist und gibt Hinweise, wo Verbesserungen in dieser Zusammenarbeit nötig wären.

Die Zukunft der Depressionsforschung wird von Florian Holsboer herausgestellt. Er beschreibt in seinem Manuskript, welche herausragende Rolle neurowissenschaftliche Erkenntnisse anstelle von künstlichen diagnostischen Kategorien für die Diagnostik von psychischen Erkrankungen spielen könnten. Es wird auch die Vision einer personalisierten Depressionstherapie entworfen, die mithilfe von Gentests und Biomarkern spezifisch und auch schon präventiv, vor Beginn der ersten Symptome, durchgeführt werden könnte. Schließlich werden Implikationen für das Gesundheitssystem diskutiert.

Ziele und Möglichkeiten, Leistungen und Versagen der Psychiatrie und Nervenheilkunde sind stets vom gesellschaftlichen und politischen Kontext abhängig. Somit besteht für unser Fach auch die besondere Gefahr schuldhafter Verstrickungen. Die DGPPN hat eine unabhängige Kommission beauftragt, um die Geschichte der psychiatrischen Fachgesellschaft und der Psychiater im sog. „Dritten Reich“ aufzuarbeiten. Dieses Thema wird ein weiterer Schwerpunkt des Kongresses sein. Zwei Arbeiten in diesem Heft beinhalten historische Analysen zu diesem Thema.

Volker Rölcke gibt in seinem Beitrag einen Überblick über den aktuellen historischen Kenntnisstand der Psychiatrie im Nationalsozialismus. Er beschreibt, wie die jüngere historische Forschung zentrale Mythen in Frage stellt, die jahrelang das Bild der Medizin im Nationalsozialismus bestimmt haben, wie beispielsweise die Annahme, dass die medizinischen Verbrechen die Tat weniger Nazi-Ärzte gewesen seien. Die Mythen werden im Hinblick auf die neuen medizinhistorischen Erkenntnisse diskutiert. Es werden Implikationen für die heutige Zeit erörtert.

Gerrit Hohendorf und Kollegen berichten schließlich über die sog. „,T4 Aktion“, bei der 1941-1943 während des Nationalsozialismus ca. 70.000 psychiatrische Patienten ermordet wurden. Die Autoren beschreiben den Umgang mit psychisch Kranken zu dieser Zeit. Anhand indivi- dueller Lebensgeschichten wird über die Sterilisation psychisch Kranker zur „Verhütung des kranken Nachwuchses“ sowie das Gedankengut der Verantwortlichen berichtet. Mithilfe von Krankenakten war es den Autoren möglich, die Selektionskriterien für Sterilisations- und Ermordungsopfer näher zu beleuchten. Dabei zeigte sich, dass Produktivität in der Arbeitsleistung das wichtigste Selektionskriterium der Opfer war.

Wir würden uns sehr freuen, wenn Sie unser Interesse sowohl für die interdisziplinäre Psychiatrie wie auch die historische Aufarbeitung der Psychiatriegeschichte als besonderen Schwerpunkt in diesem Jahr teilen würden.

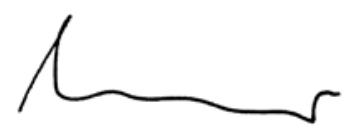

Prof. Dr. Frank Schneider

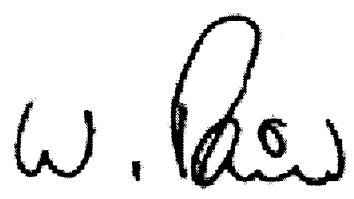

Prof. Dr. Wolfgang Maier

\section{Korrespondenzadresse}

Prof. Dr. F. Schneider

Klinik für Psychiatrie und Psychotherapie, RWTH Aachen University

Pauwelsstraße 30, 52074 Aachen

fschneider@ukaachen.de

\section{Stiftung Nordlicht vergibt Auslandsstipendien an Schüler und Erwachsene}

Jugendliche und Erwachsene, die gerne ein Auslandsprogramm absolvieren möchten, die damit verbundenen Kosten aber nicht aus eigener Kraft aufbringen können, haben noch bis zum 30. November 2010 die Möglichkeit, sich für das Nordlicht-Stipendium zu bewerben.

Entscheidend für die Vergabe eines Stipendienplatzes der im Jahre 2004 ins Leben gerufenen Stiftung Nordlicht, ist vor allem das soziale Engagement der Bewerber. Dazu zählen insbesondere Aktivitäten zur Förderung der internationalen Verständigung, aber auch die Mitarbeit in einer Gemeinde, an einer Schülerzeitung, im Alten- oder Behindertenheim oder aktive Nachbarschaftshilfe. Als Belohnung locken verschiedene Programme, die frei zur Auswahl stehen: Voll- und Teilstipendien für diverse Schüleraustauschprogramme in viele Länder weltweit und ein VolunteerStipendium für einen Freiwilligendienst inklusive Flug nach Asien.

Die Kieler Austauschorganisation KulturLife stellt zusammen mit der Deutschen Kreditbank (DKB) diese Programme als Förderer zur Verfügung. Bewerben können sich junge und jung gebliebene Menschen aus der gesamten Bundesrepublik im Alter von 14 bis 65 Jahren. Erfahrungsberichte ehemaliger und aktueller Stipendiaten, nähere Beschreibungen zu den Voraussetzungen sowie die Bewerbungsunterlagen sind als Download unter www.nordlichtstipendium.de zu finden. Bewerbungsschluss ist der 30. November 2010.

Quelle: Stiftung Nordlicht Stipenidum (Kiel) 